\title{
Adjusting Preoperative Risk Models of Post-Heart Transplant Survival to a European Cohort in the Age of a New Cardiac Allocation Score in Europe
}

\author{
Tarik-Alp Sargut, ${ }^{1}$ Julia Stein, ${ }^{1}$ Panagiotis Pergantis,,${ }^{1,2}$ Christoph Knosalla, MD,${ }^{1,2}$ Jan Knierim, MD, ${ }^{1,2}$ \\ Manfred Hummel, MD, ${ }^{3}$ Volkmar Falk, MD, ${ }^{1,2,4}$ Felix Schoenrath, MD ${ }^{1,2}$ \\ ${ }^{1}$ Department of Cardiothoracic and Vascular Surgery, German Heart Center Berlin, Berlin, Germany; ${ }^{2}$ DZHK (German \\ Centre for Cardiovascular Research), partner site, Berlin, Germany; ${ }^{3}$ Paulinenkrankenhaus Berlin, Berlin, Germany; \\ ${ }^{4}$ Department of Cardiothoracic Surgery, Charité - Universitätsmedizin Berlin, corporate member of Freie Universität Berlin, \\ Humboldt-Universität zu Berlin, and Berlin Institute of Health, Berlin, Germany
}

\section{ABSTRACT}

Background: Several risk models target the issue of posttransplant survival, but none of them have been validated in a large European cohort. This aspect is important, in a time of the planned change of the Eurotransplant allocation system to a scoring system.

Materials and Methods: Data of 761 heart transplant recipients from the Eurotransplant region with a total followup of 5027 patient-years were analyzed. We assessed 30-day to 10 -year freedom from graft failure. Existing posttransplant mortality risk models, IMPACT, MELD-XI, and Columbia risk stratification score (RSS) were evaluated. A new risk model was created, using the variables from univariate and multivariate analysis: CABDES score (coronary artery disease, donor age, use of a biventricular assist device [BVAD], diabetes mellitus, estimated glomerular filtration rate [eGFR], sex). The predictive accuracy was compared with the existing risk scores, with a focus on LVAD patients.

Results: Thirty-day, 1-year, 5-year, and 10-year rates of freedom from graft failure were $78.3 \% \pm 1.5 \%, 68.8 \% \pm$ $1.71 \%, 59.1 \% \pm 1.8 \%$ and $44.1 \% \pm 1.9$, respectively. The 1-year incidence of graft failure varied from $14.1 \%$ to $50 \%$ (RSS), from $22.9 \%$ to $57.1 \%$ (IMPACT), and from $24.9 \%$ to $42.6 \%$ (MELD-XI). Our newly adjusted risk score showed an improved area under the curve (AUC) of 0.69 (95\% CI 0.64-0.72) with better discrimination in the intermediate to moderate risk cohort (CABDES score).

Conclusion: IMPACT, MELD-XI, and RSS were suitable to predict posttransplant graft failure only in high- and lowrisk cohorts. CABDES score might be an alternative scoring system, with donor age and eGFR being the strongest predictors. Implementation of the IMPACT score within the new Eurotransplant cardiac allocation score for patient prioritization for heart transplantation should be reevaluated.

Received September 9, 2018; accepted November 9, 2018.

Correspondence: Felix Schoenrath, MD; Department of Cardiothoracic and Vascular Surgery, German Heart Center Berlin, Augustenburger Platz 1, 13353 Berlin, Germany; +49-30-4593-2085; fax +49-30-4593-2100 (e-mail:schoenratb@dbzb.de).

\section{INTRODUCTION}

Organ allocation for heart transplantation is one of the most difficult procedures in modern cardiology. Several risk models target the issue of predicting posttransplant survival, the most widely used score systems being the Columbia risk stratification score (RSS) [Hong 2011] and the Index for Mortality Prediction After Cardiac Transplantation (IMPACT) [Weiss 2011]. RSS was introduced by Hong in 2011 after evaluating UNOS patient data from 2001 to 2007. Strongest predictors for 1-year graft failure were extracorporeal right ventricular assist device (ec RVAD), extracorporeal membrane oxygenation (ECMO), renal failure, extracorporeal left ventricular assist device (ec LVAD), total artificial heart (TAH), and advanced age. IMPACT was introduced by Weiss, also in 2011, and based on UNOS data from 1997 to 2008. Strongest negative predictors in the established model for 1-year graft failure were impaired renal function, congenital etiology of heart failure, mechanical ventilation prior to transplantation, temporary circulatory support, and continuous flow VADs (Jarvik [Jarvik Heart Inc], DeBakey VAD ${ }^{\circledR}$ [MicroMed Technology], VentrAssist [Ventracor]) with the exception of HeartMate II® devices. In 2013, IMPACT was

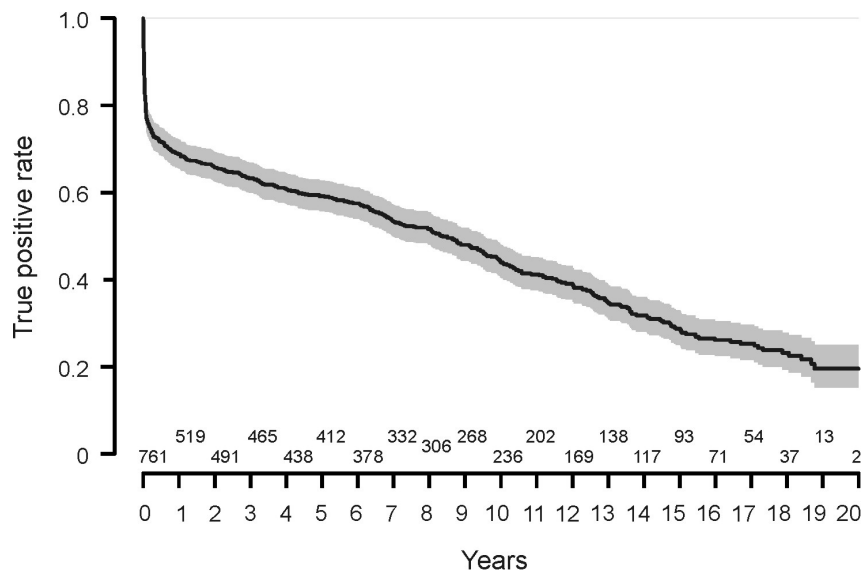

Figure 1. Overall freedom from graft failure. Numbers immediately above the $x$-axis indicate patients at risk. Gray areas around the survival curve indicate the confidence interval. 


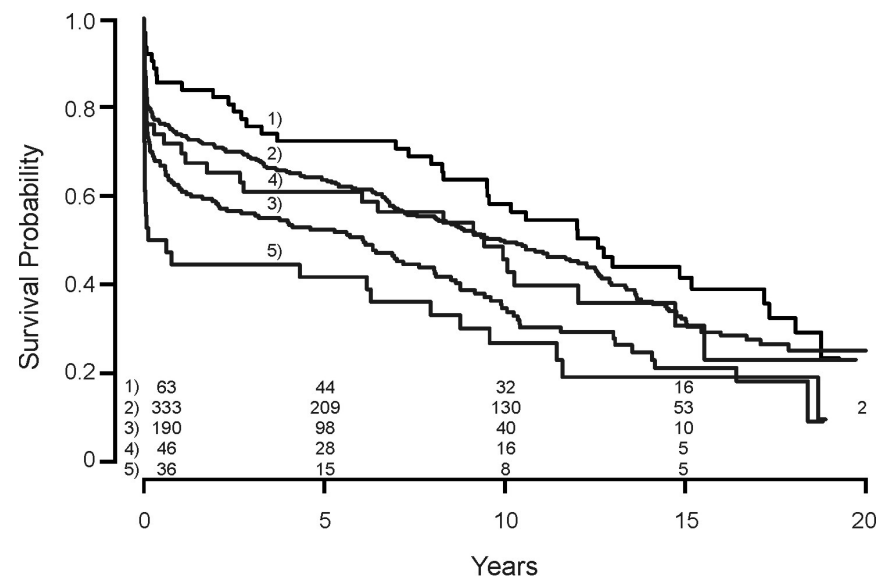

Figure 2. Freedom from graft failure stratified by the 5 strata of the RSS. 1, low risk; 2, intermediate risk; 3, moderate risk; 4 , elevated risk; 5 , high risk. Numbers immediately above the $x$-axis indicate patients at risk.

validated by using the ISHLT data from 2001 to 2011 [Kilic 2013]. Of course this data is mainly driven by UNOS data, and data from the Eurotransplant region accounts only for approximately $15 \%$ of this data (ISHLT database, 2014 Jul 1 to 2015 Jun 30: 4333 heart transplants; Eurotransplant database, 2014: 634 heart transplants). In 2013, IMPACT was used by Smits et al [Smits 2013] to evaluate 189 patients who underwent heart transplantation in the Eurotransplant region, with a follow-up of 12 weeks. Another score used for survival after heart transplantation is MELD-XI. As shown in 2016 by Szygula et al in 87 patients, creatinine and bilirubin values were able to predict 1 -year survival after heart transplantation [Szyguła-Jurkiewicz 2016].

Because none of these scoring systems had been validated in a large, contemporary homogenous European cohort in a long-term approach, we conducted our analysis. This aspect seems of utmost importance, not only for preoperative patient selection in each transplant center, but also on a European level the reliable forecast of posttransplant survival and therefore allocation of donor organs efficiently, in a time of the planned switch of the Eurotransplant allocation system from a binary system (prioritized-high urgent [HU] status-versus nonprioritized-transplantable [T] status) to a scoring system (cardiac allocation score [CAS]) similar to the lung allocation score (LAS) for lung transplantation [Smits 2011].

\section{MATERIALS AND METHODS}

\section{Study Population}

The patient data for this retrospective single center study were provided by our institutional transplant registry. We analyzed the data of 761 patients aged 18 years and older having undergone orthotopic heart transplantation during the time period from 1996 to 2015. Exclusion criteria were simultaneous transplantation of heart and lung, cardiac retransplantation, and age under 18 years. Follow-up time was 5.9

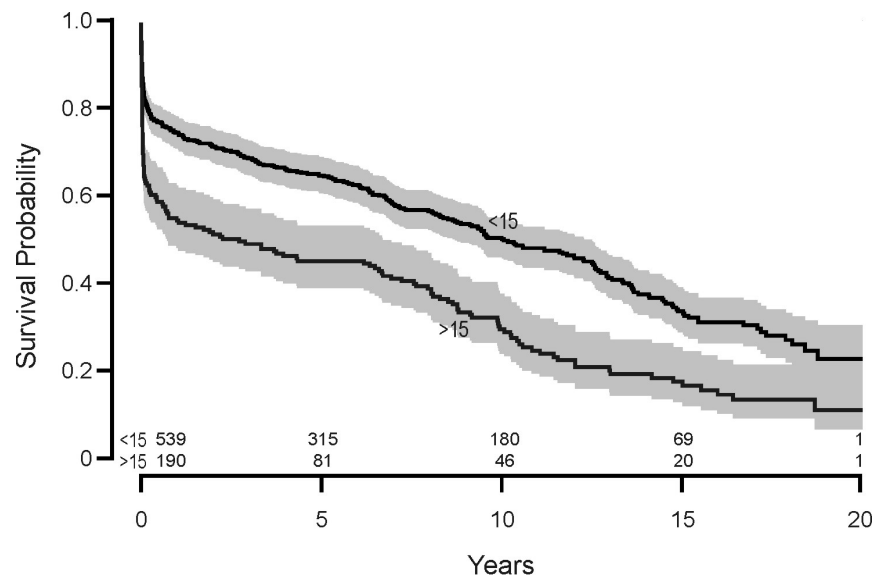

Figure 3. Freedom from graft failure stratified by the MELD XI score. $<15$, low risk; $>15$, high risk. Numbers immediately above the $x$-axis indicate patients at risk.

years (range 0-19 years) with a total of 5027 patient-years. Our patient data was provided through 2016. For all patient characteristics the last documented follow-up available in our records was used. A specific vote of the ethics committee of the Charité - Universitätsmedizin Berlin for this project based on a retrospective analysis of data resulting solely from standard of care therapy within our center was not required. Collection and analysis of clinical data was performed according to the data protection act of Berlin.

Steroid and antibody therapy was used as the induction regimen. Afterwards the posttransplantation immunosuppressive regimen consisted of 3 drugs: mycophenolate mophetil, a calcineurin inhibitor (cyclosporine or tacrolimus), and prednisone. During the following course the regimen was further individualized, including treatment with everolimus, if indicated. Statins were used in all patients able to tolerate this therapy.

\section{Risk Scores}

First, we assessed 30-day, 1-year, 5-year, and 10-year freedom from graft failure and performed univariate and multivariate mortality risk analyses.

Second, we compared different risk models including IMPACT, MELD-XI, and RSS. IMPACT is a 50-point scoring system incorporating 12 recipient-specific variables [Weiss 2011]. MELD-XI is defined by the following formula: MELD-XI = $5.11 \ln$ (bilirubin $)+11.76 \ln ($ creatinine $)+9.44$. Creatinine and total bilirubin were measured as milligram/ deciliter [Heuman 2007]. RSS analyzed pretransplant recipient and donor characteristics and predicted graft failure at 1 year [Hong 2011]. With our data, IMPACT was feasible for 732 (96.2\%), MELD-XI for 729 (95.7\%), and RSS for 669 $(87.9 \%)$ patients of our cohort.

Third, we adjusted the risk models with different variables by using a multivariate logistic regression model to achieve an optimal predictive value. Therefrom we created a new risk score and compared predicted long-term freedom from graft failure to data provided by IMPACT, MELD-XI, and RSS.

Fourth, a further analysis was performed with a special 


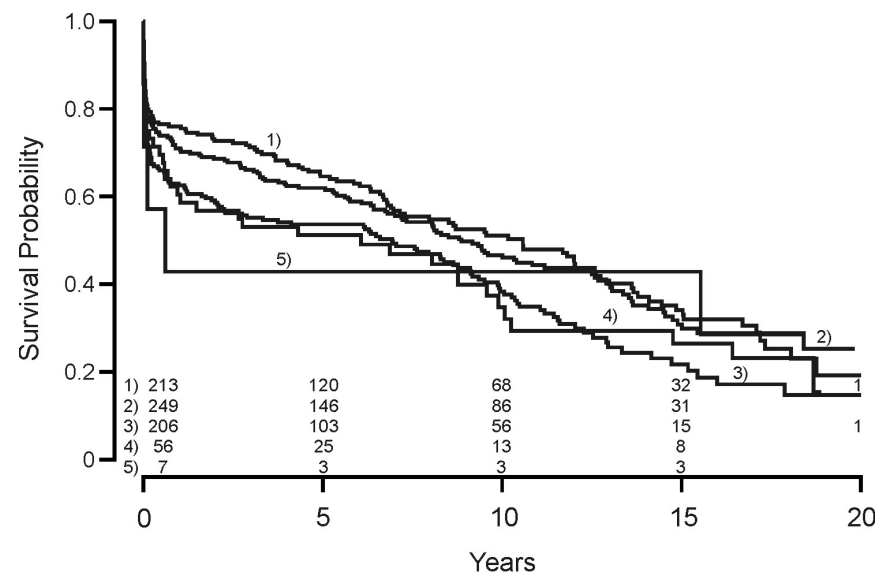

Figure 4. Freedom from graft failure stratified by the 5 strata of the IMPACT score. 1, low risk, 0-2 points; 2, intermediate risk, 3-5 points; 3, elevated risk, 6-9 points; 4, moderate risk, 10-14 points; 5, high risk, $>14$ points. Numbers immediately above the $x$-axis indicate patients at risk for each stratum. Gray areas around the survival curve indicate the confidence interval.

focus on LVAD patients.

\section{Statistical Analysis}

Continuous variables are presented as mean (standard deviation). For categorical variables, absolute and relative frequencies are given. Comparisons between groups are analyzed by Student $t$ test or Wilcoxon test for continuous and $\chi^{2}$ for categorical variables. Kaplan-Meier curves were calculated for all risk groups to evaluate the scores' ability to predict freedom from graft failure. Patients lost to follow-up or having been without graft failure after one year were censored.

As a measure for the discriminating ability, the area under the curve (AUC) for both scores was calculated.

For development of the new score, all relevant risk factors of IMPACT and RSS were used, and further variables were added. To take account of the long observation time of 20 years, the year of transplantation was also evaluated. Missing values in candidate risk variables were replaced by multiple imputations. Multivariate logistic regression was used to develop a model to predict 1-year graft failure.

We considered the preoperative risk factors that were statistically significant in univariate analysis for inclusion. Continuous variables were modeled by using cubic spline with 3 knots to account for nonlinear relationships. A backward selection procedure was employed, and bootstrap resampling procedure was used to study the stability of the stepwise selection model. The optimism of the selected model was calculated by using 1000 bootstrap samples to correct the C-statistic from over-optimism [Efron 1993]. For calibration, the Hosmer-Lemeshow test [Hosmer 1997] and Brier score [Brier 1950] were calculated. The odds ratios of the final logistic regression model were used to assign weights for each of these risk factors. The explanatory power of the final model was assessed by Akaike information criterion (AIC), likelihood ratio tests, and the AUC.

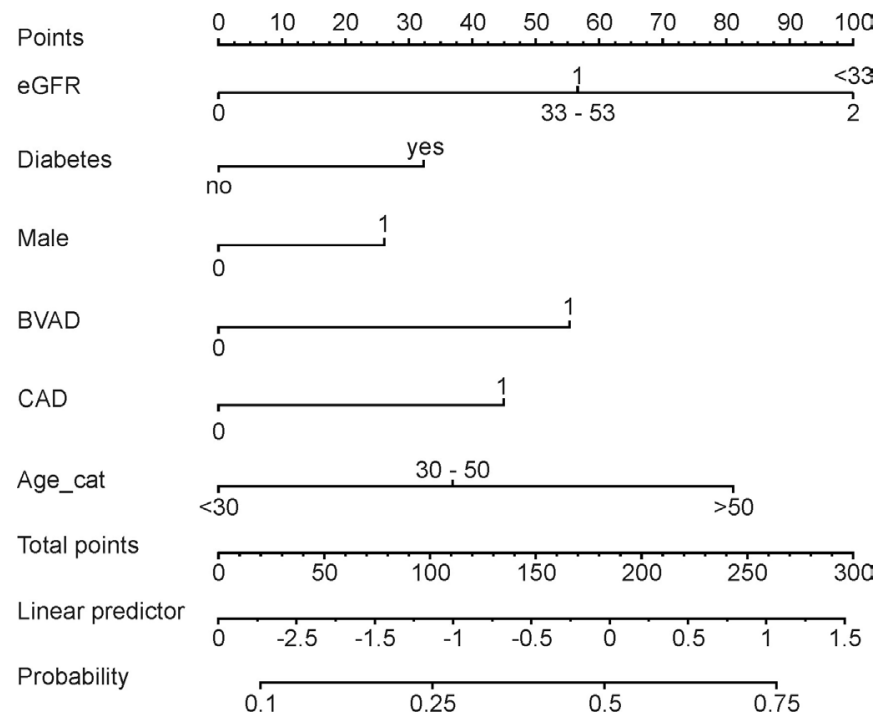

Figure 5. Nomogram to calculate predicted event-free 1-year survival. For example, a male patient with eGFR of $50 \mathrm{~mL} / \mathrm{min}$, no diabetes, no BVAD, CAD, and a 20-year-old donor heart has total points of $27+27$ $+0+0+45+0$. This corresponds to predicted 1-year graft failure of $25 \%$. Age_cat, age category.

The result of the model procedure is also presented as a nomogram, which makes it possible to judge the relative importance of each predictor by the number of points attributed over the range of the predictor and also gives estimates of the probability of graft-failure-free 1-year survival.

The R statistical package, Hmisc, MASS, and rms libraries were used for analysis [R Core Team 2013].

\section{RESULTS}

Descriptive characteristics of the patient cohort and univariate and multivariate analyses of risk factors are displayed in the Table. The mean age was 50 (range 18-70) years with $16 \%$ female patients.

Thirty-day, 1-year, 5-year, and 10-year rates of freedom from graft failure were $78.3 \% \pm 1.5 \%, 68.8 \% \pm 1.71 \%, 59.1 \%$ $\pm 1.8 \%$ and $44.1 \% \pm 1.9 \%$, respectively (see Figure 1 ).

The 1-year incidence of graft failure in accordance with the 5 RSS strata was $14.1 \%, 25.2 \%, 37.4 \%, 28.3 \%$, and $50 \%$.

The 1-year freedom from graft failure using a cutoff of 15 points in MELD-XI ranged from $57.4 \%($ MELD-XI $>15)$ to 75.1\% (MELD-XI <15).

The 1-year incidence of graft failure stratified by 3 -point increments of IMPACT showed incidence rates varying from $22.9 \%$ ( $0-2$ points) to $57.1 \%$ ( $>15$ points).

AUC was 0.59 (95\% CI 0.54-0.64) for IMPACT, 0.60 (95\% CI 0.55-0.65) for MELD-XI, and 0.62 (95\% CI 0.57$0.66)$ for RSS. Long-term discrimination between the different risk strata for RSS, MELD-XI, IMPACT is shown in Figures 2-4.

Multivariate analysis was used to adjust a new risk score (CABDES score). With the variables coronary artery disease, donor age, use of a biventricular assist device (BVAD), 


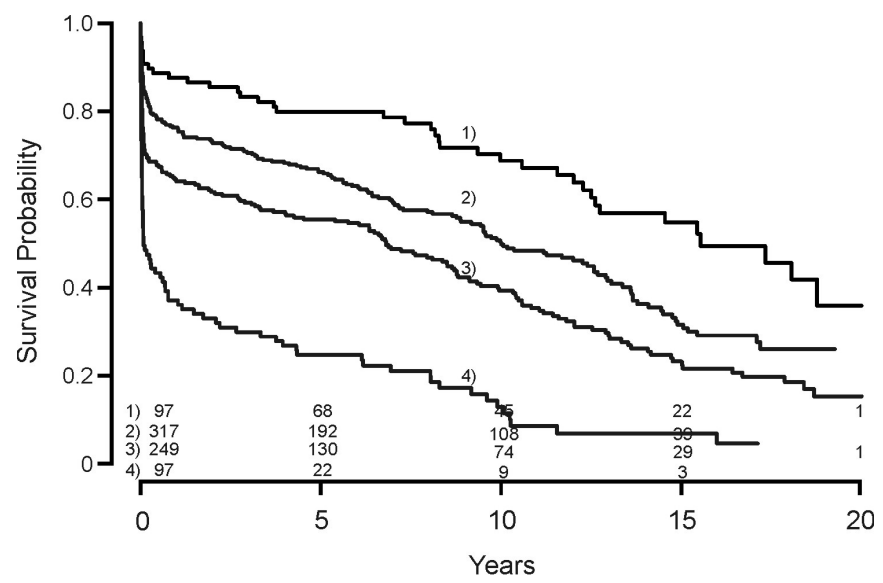

Figure 6. Freedom from graft failure stratified by the 4 strata of the CABDES score. 1, low risk, 0-2.9 points; 2 , intermediate risk, 3-4.9 points; 3, moderate risk, 5-6.9 points; 4 , high risk, $>6.9$ points. Numbers immediately above the $x$-axis indicate patients at risk for each stratum.

diabetes mellitus, estimated glomerular filtration rate (eGFR), sex, the new score (CABDES score) was created. Additional information about 2 of the variables that were used is the following: BVAD as bridge to transplant therapy, pretransplant diabetes mellitus. Donor age and eGFR appeared to be strong predictors and were almost always (99\% and $100 \%$, respectively) selected when stepwise selection procedure was repeated in bootstrap samples. To calculate the predicted event-free 1-year survival, a normogram (see Figure 5) could be used. No further improvement was achieved after adding further variables which were significant in the univariate analysis.

Hosmer-Lemeshow test showed an adequate fit with $P=.7$, and Brier score was $0.189(P<.001)$. With 1000 bootstrap replicates, the estimated optimism is 0.033 , and the optimism-corrected C-statistic of 0.67 showed slightly improved discrimination compared to the other scores. Odds ratios for the variables used are displayed in the Table.

The CABDES score showed an AUC of 0.69 (95\% CI $0.64-0.72)$. It ranged from 0 to $11.4($ mean $=4.8)$, with a $41 \%$ increase in graft failure risk per unit risk score (odds ratio 1.41 (95\% CI 1.30-1.53), $P<.001$ ). To predict risk of graft failure, 4 strata were used: 0-2.9 points, 3-4.9 points, 5-6.9 points, and $>7$ points, resulting in a 1 -year incidence of graft failure of $12.4 \%, 22.6 \%, 34.9 \%$, and $59.8 \%$, respectively. Long-term discrimination between the strata is displayed in Figure 6.

No lack of discrimination in the subset of patients with currently still-used LVADs (HeartMate II, HeartWare®) and a good stratification were seen with the new score (Figure 7).

\section{DISCUSSION}

Adequate preoperative risk stratification is of great importance in all fields of surgery to achieve optimal postoperative survival. It is of utmost importance in the area of solid organ transplantation, with a shortage of resources (donor organs).

In contrast to our data and the current ISHLT data [Lund

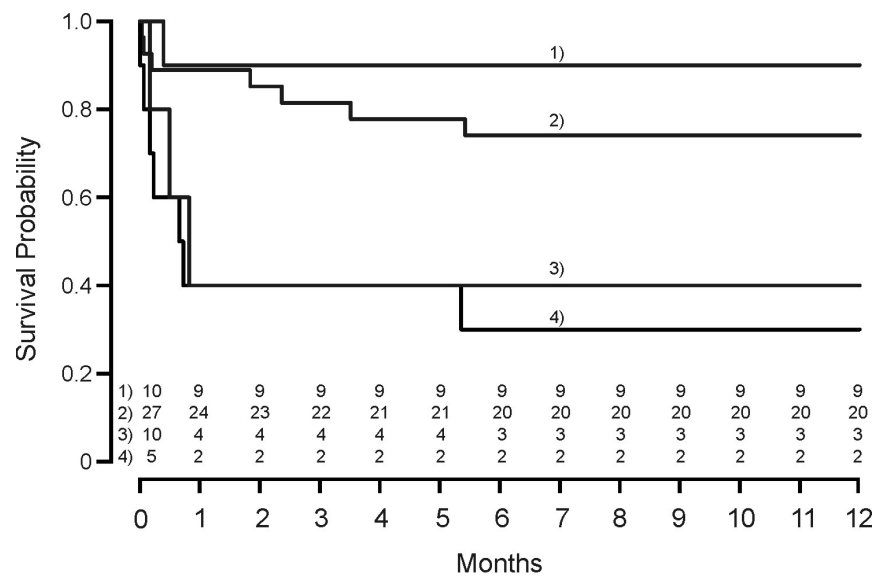

Figure 7. Freedom from graft failure stratified by the 4 strata of the CABDES score for LVAD patients. 1, low risk, 0-2.9 points; 2 , intermediate risk, 3-4.9 points; 3, moderate risk, 5-6.9 points; 4 , high risk, $>6.9$ points. Numbers immediately above the $x$-axis indicate patients at risk for each stratum.

2016], female sex was stated as a risk factor in IMPACT. Ischemic origin of heart failure was a relevant risk factor in our cohort, as it was in [Weiss 2011] and in the current ISHLT data although not in the RSS [Hong 2011]. Renal failure and older donor age are well-established risk factors [Hong 2011; Weiss 2011] and were the strongest predictors in our cohort. In contrast to various other risk scores (eg, STS score, EuroSCORE II) in cardiovascular medicine, diabetes mellitus is not mentioned as a risk factor in RSS and did not show significance in $\operatorname{IMPACT}(P=.08)$ [Hong 2011; Weiss 2011], but was previously established as a risk factor [Higgins 2009] after heart transplantation (confirmed recently [Foroutan 2018]), and was a relevant risk factor in our cohort. Other risk factors, such as IABP [Weiss 2011], ECMO, or ventilation prior to transplantation [Hong 2011] did not show significance in our estimation, which might be influenced by the small numbers of these characteristics in our analysis. This is of course a limitation of a single-center trial; on the other hand this patient population unfortunately does not reflect the patient cohort currently receiving transplantation within the Eurotransplant area, because estimated waiting time exceeds the time span of these short-term interventions.

LVAD did not place the patient at an additional risk for graft failure in our cohort. This is consistent with contemporary ISHLT data [Lund 2016] and a recently published metaanalysis [Foroutan 2018]. Older scoring systems place patients with LVAD support in a higher risk group, with this being one of the findings of the first 2 IMPACT publications [Weiss 2011; Kilic 2013]. When evaluated on a contemporary VAD cohort [Smits 2013], IMPACT failed to predict survival. RSS was never evaluated in patients with contemporary VAD systems, which significantly limits the clinical benefit of this model, even more, with the ongoing development and increasing use of VADs for treatment of end-stage heart failure [Lund 2016]. In contemporary ISHLT data [Lund 2016], this subset counts for up to $41.3 \%$ with a relevant increase during the past decade. This has even more clinical implications in the advent of a changing allocation system. Scoring 
Descriptive Characteristics and Univariate and Multivariate Analysis*

\begin{tabular}{|c|c|c|c|c|c|c|}
\hline \multicolumn{7}{|l|}{ Demographics } \\
\hline Age >60 y, n (\%) & $123(16.16)$ & $45(19.7)$ & $78(14.7)$ & .08 & $1.42(0.9-2.1)$ & \\
\hline Male, n (\%) & $636(83.6)$ & $199(86.9)$ & $437(82.1)$ & .104 & $0.69(0.4-1.1)$ & $1.42(0.9-2.3)$ \\
\hline 1996-2000, n (\%) & $279(36.7)$ & $80(34.9)$ & 199 (37.4) & .365 & & \\
\hline 2001-2005, n (\%) & $240(31.5)$ & $66(28.8)$ & $174(20.1)$ & & $0.94(0.63-1.39)$ & \\
\hline 2006-2010, n (\%) & $165(21.7)$ & $58(25.3)$ & $107(20.1)$ & & $1.35(0.89-2.04)$ & \\
\hline 2011-2014, n (\%) & $77(10.1)$ & $25(10.9)$ & $52(9.8)$ & & $1.20(0.70-2.06)$ & \\
\hline \multicolumn{7}{|l|}{ Etiology } \\
\hline \multicolumn{7}{|l|}{ Mechanical support } \\
\hline LVAD, n (\%) & $170(22.3)$ & $48(21.0)$ & $122(22.9)$ & .163 & $0.95(0.6-1.4)$ & \\
\hline BVAD, n (\%) & $85(11.2)$ & $33(14.4)$ & $52(9.8)$ & & $1.54(1.0-2.5)$ & $2.13(1.3-3.5)$ \\
\hline No VAD, n (\%) & $506(66.5)$ & $148(64.6)$ & $358(67.3)$ & & - & \\
\hline TAH, n (\%) & $10(1.3)$ & $5(2.2)$ & $5(0.9)$ & & & \\
\hline \multicolumn{7}{|l|}{ Comorbidities/others } \\
\hline BMI, mean (SD) & $25.04(3.9)$ & $25.65(4.1)$ & $24.78(3.8)$ & .005 & & \\
\hline $\mathrm{BMI}<20, \mathrm{n}(\%)$ & $65(8.7)$ & $18(8.0)$ & $47(9.1)$ & & - & \\
\hline BMI 20-30, n (\%) & $596(80.1)$ & $170(75.6)$ & $426(82.1)$ & & $1.0(0.6-1.8)$ & \\
\hline eGFR 33-53, n (\%) & $200(26.3)$ & $77(33.6)$ & $123(23.1)$ & & $1.99(1.4-2.8)$ & $2.1(1.5-3.1)$ \\
\hline eGFR <33, n (\%) & $73(9.6)$ & $35(15.3)$ & $38(7.1)$ & & $2.92(1.8-4.8)$ & $3.80(2.2-6.5)$ \\
\hline $\begin{array}{l}\text { Previous cardiac } \\
\text { surgery }\end{array}$ & $434(57.0)$ & $137(59.8)$ & $297(55.8)$ & .307 & $1.17(0.8-1.6)$ & \\
\hline Diabetes, n (\%) & 137 (18) & $53(21.1)$ & $84(15.8)$ & .015 & $1.61(1.1-2.4)$ & $1.55(1.1-2.3)$ \\
\hline Acuity & & & & .671 & & \\
\hline Ambulant, n (\%) & $425(55.8)$ & $125(54.6)$ & $300(56.4)$ & .648 & - & \\
\hline Hospitalized, n (\%) & $332(43.6)$ & $102(44.5)$ & $230(43.2)$ & & $1.1(0.8-1.5)$ & \\
\hline Ventilated, n (\%) & $4(0.5)$ & $2(0.9)$ & $2(0.4)$ & & $2.4(0.3-17.2)$ & \\
\hline Blood type & & & & .893 & & \\
\hline O, n (\%) & $247(32.5)$ & $73(31.9)$ & $174(32.8)$ & & - & \\
\hline A, n (\%) & $330(43.5)$ & $103(45.0)$ & $227(42.8)$ & & $1.08(0.76-1.55)$ & \\
\hline B, n (\%) & $118(15.5)$ & $36(15.7)$ & $82(15.5)$ & & $1.05(0.6-1.17)$ & \\
\hline$A B, n(\%)$ & $64(8.4)$ & $17(7.4)$ & $47(8.9)$ & & $0.86(0.5-1.6)$ & \\
\hline
\end{tabular}


Table 1. Descriptive Characteristics and Univariate and Multivariate Analysis*

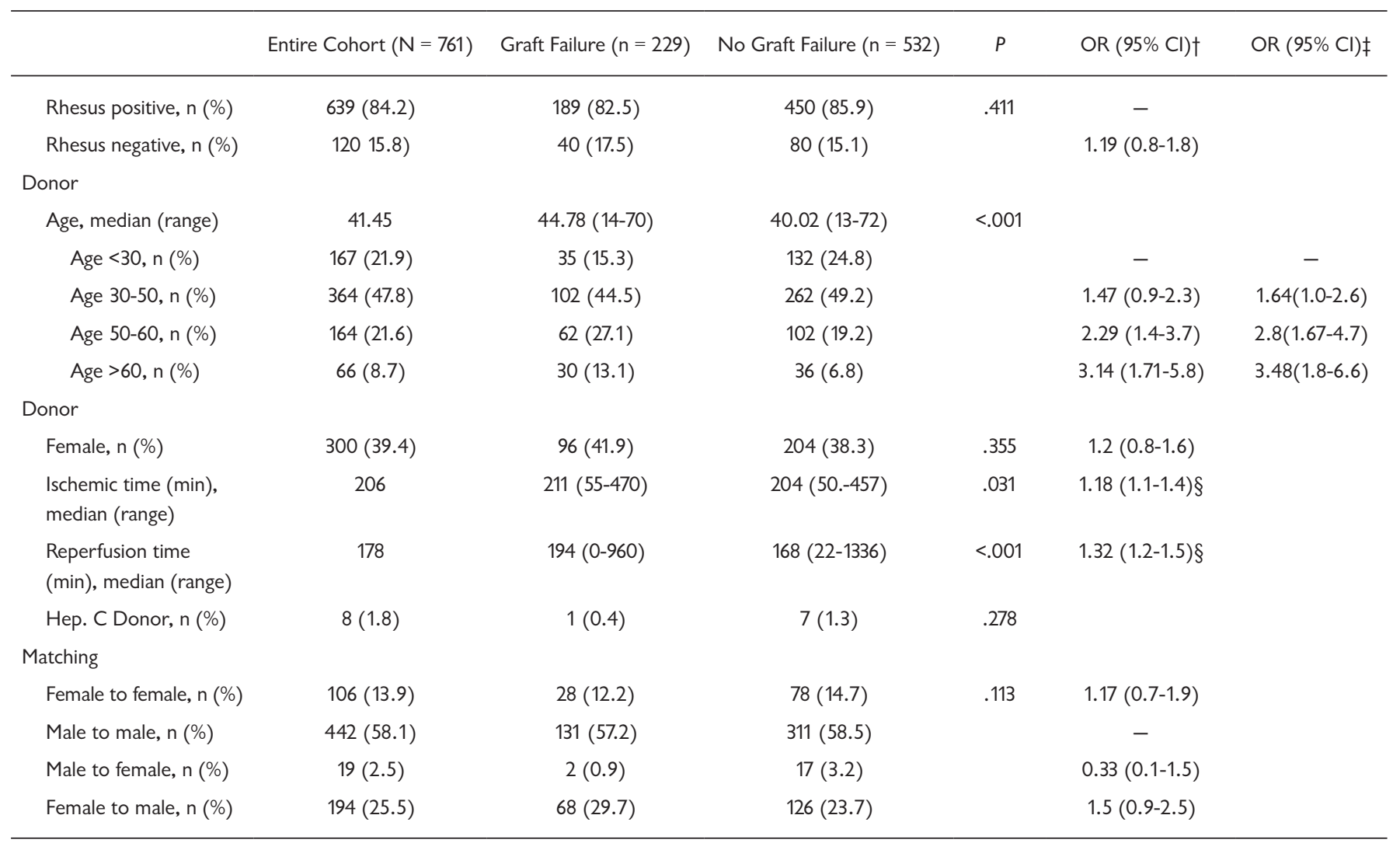

*DCMP, dilated cardiomyopathy; BVAD, biventricular assist device(s) including the following: EXCOR® (Berlin Heart), SynCardia CardioWest ${ }^{\mathrm{TM}}$; LVAD, left ventricular assist device(s) including the following: INCOR® (Berlin Heart), Jarvik 2000® (Jarvic Heart), Heartmate II (Thoratec Corp.), HeartWare, Novacor ${ }^{\circledR}$ (World Heart Inc.), DeBakey VAD® (MicroMed Technology Inc.); VAD, ventricular assist device; eGFR, estimated glomerular filtration rate. †Univariate.

$\ddagger$ Multivariate.

$\S$ Per hour.

patients with an LVAD at a higher (IMPACT-driven) risk (because LVAD therapy is a risk factor within IMPACT) could result in a lower probability of transplantation, because patients with a higher risk of posttransplant mortality should not be prioritized with the new CAS [Smits 2013]. IMPACT, for posttransplant survival, and the Seattle Heart Failure Model, for waitlist mortality, have been announced to be the 2 important factors of the new CAS [Smits 2013].

In our overall cohort, both scoring systems (IMPACT and RSS) were able to predict long-term graft failure only in the high- and low-risk cohorts. A completely different approach compared to complex scoring systems is the MELD-XI. With only 2 very easily assessed preoperative variables, it was possible to predict short-term survival in a small cohort [SzygułaJurkiewicz 2016] as well as short- and long-term freedom from graft failure in our cohort. AUC was comparable to both, IMPACT and RSS. In the clinically most relevant intermediate risk cohort, these scoring systems failed to predict graft failure. The variables that were used for our adjusted score (CABDES) predicted short- and long-term freedom from graft failure better, even in the intermediate cohort, and did not show a lack of discrimination in the important subset of LVAD patients, with consistency for contemporary devices (HeartMate II and HeartWare).

RSS and IMPACT were primarily evaluated with UNOS data. But, because there are relevant differences in listing modalities between different areas (eg, different prioritization models) and other short- and long-term treatment options with MCS are influenced by reimbursement and local availability, comparison between the USA and Eurotransplant is challenging [de By 2015; Kirklin 2017; Reineke 2017].

Especially in LVAD patients, owing to different prioritization rules (LVAD patients are automatically given $1 \mathrm{~B}$ status and are allowed a 30-day $1 \mathrm{~A}$ period to avoid device complications in the USA compared with prioritization only if complications occur, in the Eurotransplant region) [Lund 2016], patient cohorts having received transplantations are completely different.

This might also be one rationale for the inconsistent literature [Hong 2011; Weiss 2011; Szyguła-Jurkiewicz 2016; 
Foroutan 2018] for the posttransplant survival rates after extracorporeal BVAD support, which was a relevant risk factor for graft failure in our cohort. Because especially in these systems the complication rate is high [VanderPluym 2018] and therefore the longer support is necessary, the more likely relevant problems occur and influence longitudinal transplant survival.

\section{CONCLUSION}

In conclusion modeling and evaluation of a score that reflects the situation within the Eurotransplant region is essential.

Therefore, the presented parameters might be of additive value in the further evaluation process of the CAS.

\section{STUDY LIMITATIONS}

Like any retrospective review this study has limitations. It is a single-center cohort of selected patients, recruited and followed up in our center. Significance of less common etiologies and characteristics might be underestimated owing to small numbers. To overcome the disadvantage of modeling a score in a single-center cohort at least partially, we used bootstrap resampling to correct the C-statistic from over optimism [Efron 1993]. After correction, the AUC had to be adjusted only marginally ( 0.69 to 0.67 ). Because not all modeling effects can be considered in a bootstrap procedure, a further external validation with more recent patients and in other settings would be required.

\section{ACKNOWLEDGMENT}

We thank Anne Gale for editorial assistance.

\section{REFERENCES}

Brier GW. 1950. Verification of forecasts expressed in terms of probability. Mon Weather Rev 78:1-3.

de By TM, Mohacsi P, Gummert J, et al. 2015. The European Registry for Patients with Mechanical Circulatory Support (EUROMACS): first annual report. Eur J Cardiothorac Surg 47:770-6; discussion 776-7.

Efron B, Tibshirani RJ. 1993. An introduction to the Bootstrap. New York: Chapman and Hall.

Foroutan F, Alba AC, Guyatt G, et al. 2018. Predictors of 1-year mortality in heart transplant recipients: a systematic review and meta-analysis. Heart 104:151-60.
Heuman DM, Mihas AA, Habib A, et al. 2007. MELD-XI: a rational approach to "sickest first" liver transplantation in cirrhotic patients requiring anticoagulant therapy. Liver Transpl 13:30-7.

Higgins J, Pflugfelder PW, Kostuk WJ. 2009. Increased morbidity in diabetic cardiac transplant recipients. Can J Cardiol 25:e125-9.

Hong KN, Iribarne A, Worku B, et al. 2011. Who is the high-risk recipient? Predicting mortality after heart transplant using pretransplant donor and recipient risk factors. Ann Thorac Surg 92:520-7.

Hosmer DW, Hosmer T, Le Cessie S, Lemeshow S. 1997. A comparison of goodness-of-fit tests for logistic regression models. Stat Med 16:965-80.

Kilic A, Allen JG, Weiss ES. 2013. Validation of the United States-derived Index for Mortality Prediction After Cardiac Transplantation (IMPACT) using international registry data. J Heart Lung Transplant 32:492-8.

Kirklin JK, Pagani FD, Kormos RL, et al. 2017. Eighth annual INTERMACS report: special focus on framing the impact of adverse events. J Heart Lung Transplant 36:1080-6.

Lund LH, Edwards LB, Dipchand AI, et al. 2016 The Registry of the International Society for Heart and Lung Transplantation: thirty-third adult heart transplantation report-2016; focus theme: primary diagnostic indications for transplant. J Heart Lung Transplant 35:1158-69.

R Core Team. 2013. R: a language and environment for statistical computing. Vienna (Austria): R Foundation for Statistical Computing. Available from: http://www.R-project.org/

Reineke DC, Mohacsi PJ. 2017. New role of ventricular assist devices as bridge to transplantation: European perspective. Curr Opin Organ Transplant 22:225-30.

Smits JM, de Vries E, De Pauw M, et al. 2013. Is it time for a cardiac allocation score? First results from the Eurotransplant pilot study on a survival benefit-based heart allocation. J Heart Lung Transplant 32:873-80.

Smits JM, Nossent GD, de Vries E, et al. 2011. Evaluation of the lung allocation score in highly urgent and urgent lung transplant candidates in Eurotransplant. J Heart Lung Transplant 30:22-8.

Szyguła-Jurkiewicz B, Zakliczyński M, Szczurek W, Nadziakiewicz P, Gąsior M, Zembala M. 2016. Predictive value of the model for end-stage liver disease score excluding international normalized ratio one year after orthotopic heart transplantation. Transplant Proc 48:1703-7.

VanderPluym CJ, Cedars A, Eghtesady P, et al. 2018. Outcomes following implantation of mechanical circulatory support in adults with congenital heart disease: an analysis of the Interagency Registry for Mechanically Assisted Circulatory Support (INTERMACS). J Heart Lung Transplant 37:89-99.

Weiss ES, Allen JG, Arnaoutakis GJ, et al. 2011. Creation of a quantitative recipient risk index for mortality prediction after cardiac transplantation (IMPACT). Ann Thorac Surg 92:914-21. 